\title{
Inspecter en pensant l'avenir...
}

\section{Werner Bauer}

Dr méd., président de l'Institut suisse pour la formation médicale postgraduée et continue ISFM

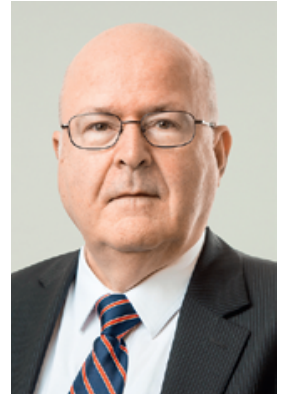

J'ai récemment participé à deux inspections de centres de formation postgraduée. Les inspections sont un instrument d'assurance qualité important pour la formation postgraduée des spécialistes. Elles sont organisées après un changement de direction à la tête d'un centre de formation, ou quand l'enquête annuelle réalisée auprès des médecins assistants révèle des problèmes. Le responsable de l'inspection est un expert de la discipline médicale concernée. Il est accompagné d'un expert non spécialiste de cette discipline, venant de l'ISFM, et d'un représentant de l'ASMAC. L'équipe se fait une image précise des conditions de formation postgraduée en parlant avec la direction de l'hôpital, avec celle de la clinique, avec les médecins cadres et, dans le plus strict anonymat, avec les médecins en formation. A partir de là, un rapport est rédigé, sur lequel le centre de formation postgraduée peut prendre position et qui contient une proposition de reconnaissance ou d'éventuelles obligations ou recommandations contraignantes. Ce rapport est transmis à la Commission des centres de formation postgraduée, qui doit prendre une décision officielle.

Plus de cent inspections ont lieu chaque année. Elles sont en bien acceptées et suscitent également l'intérêt d'observateurs étrangers. Les systèmes d'évaluation avec visite sur site sont de plus en plus répandus dans le monde. Nous constatons régulièrement qu'elles sont l'occasion de débattre du concept de formation postgraduée et de le faire évoluer.

C'est parfait. Mais indépendamment des questionnements locaux, les inspections sont aussi une source de réflexions permanentes, ainsi qu'une cause d'inquiétude. Deux questions se posent: d'une part l'équilibre entre formation et prestations, d'autre part (et de nos jours c'est bien sûr lié) le contexte économique, de plus en plus axé sur l'efficacité des établissements de formation postgraduée, avec l'augmentation de la charge administrative que cela implique.

Dans certains pays, les médecins en formation postgraduée sont payés par le Ministère de la santé ou par une organisation publique équivalente, et les centres de formation postgraduée reçoivent aussi une indemnisation pour les ressources qu'ils mettent à disposition pour la formation. Ces systèmes sont souvent accompagnés d'une supervision rapprochée et de contrôles. Notre système plus libéral applique plutôt un «enseigne- ment» dans lequel les personnes à former contribuent aux frais avec leurs prestations.

Les évolutions actuelles du système de santé remettent en question l'équilibre nécessaire entre formation et travail. La pression économique, l'obligation d'augmentation maximale de l'efficacité et l'explosion des tâches administratives menacent de plus en plus la qualité de la formation postgraduée. Lors de nombreuses inspections, le directeur d'hôpital ou de clinique décrit une augmentation des consultations ou du nombre de patients au cours des dernières années, avec une satisfaction manifeste et, de son point de vue, compréhensible. Indépendamment de tous les aspects concernant la formation, cela nous rappelle que, dans ce type de fonction, personne n'est félicité ni reconnu quand ce nombre diminue, alors que cela contribue aux économies du système de santé.

Le développement nécessaire des consultations ambulatoires et des durées de séjour plus courtes, ne cessent d'alourdir la charge des médecins en formation. Ces évolutions ont beau avoir une incidence positive sur le nombre de cas examinés, il n'en reste pas moins qu'elles présentent de plus en plus d'aspects négatifs: moins de temps pour être en contact avec les patients ou suivre une formation clinique, prédominance de l'administratif, tableaux de service qui ne permettent pas la continuité et médecins-cadres surchargés. Les programmes d'interventions chirurgicales de plus en plus chargés ont aussi des conséquences néfastes sur la formation postgraduée pratique dans les spécialités chirurgicales, les responsables de la formation ayant de moins en moins de temps pour accompagner une/un jeune collègue. Inspecter en pensant l'avenir. Nous avons sans conteste une formation postgraduée de haut niveau; mais étant donné les modifications structurelles dans le secteur de la santé, nous devons en prendre soin. Car elle assure la qualité des soins médicaux dans l'avenir. C'est pourquoi il faut trouver des moyens de soutenir et de promouvoir l'importance (qui commence déjà à s'effriter à certains endroits) de l'enseignement à tous les niveaux de la formation médicale, par rapport aux autres fonctions hospitalières et scientifiques.

Et pour reprendre les paroles de W. Osler: «The work of an institution in which there is no teaching is rarely first class» (le travail d'une institution dans laquelle on ne fournit aucun enseignement est rarement excellent). 